\title{
A Strong Standing Management Apparatus in the Confederate Threat
}

\author{
S. Kanna Babu, B.Yedukondalu
}

\begin{abstract}
In the Facilities as a Service (IaaS) elegant of cloud pc, device assets square degree on hand for apartment rate. in spite of the fact that it gives a cost practical desires to on-line machine requirements, low recollect at the leased system sources keeps human beings from exploitation it. to reduce the cost, device assets square diploma shared, i.e., there exists multi-occupancy. for the reason that correspondence systems and furthermore one of a kind machine property rectangular approach shared, it creates fitness and safety simply as protection troubles. A patron in all likelihood may not build up a legitimate co-occupant for the motive that customers square hobby mystery. The client is based upon at the Cloud provider (CP) to assign reliable co-inhabitants. but, it's in the CP's pace of intrigue that it receives most use of its assets. in this way, it lets in most co-occupancy despite the acts of clients. at some point of this paper, we often will in standard prescribe a stable call the executives system that dreams the cycle in a genuinely combine cloud to cut up within the middle of practical and furthermore dangerous customers just as allot assets in such a few way that they're doing no longer proportion assets. we frequently will in widespread show the rightness simply because the adequacy of the decided name the board framework exploitation regular and theoretical exam.
\end{abstract}

\section{INTRODUCTION}

Within the IaaS perspective of scattered laptop, computational assets are shared to restrict the cost of renting them, i.e., there exists multi-residency. as the correspondence channels except, special sources are shared, this makes guarantee simply as insurance worries. Occurrences of such issues are aspect-channel attacks, check assaults,. these well-being and security issues maintain up two or three customers from getting appropriated processing. To augmentation customer's trust finance on Cloud vendors (CP), the popularity of the CPs can be utilized as it propels customers to choose an inexpensive CP. A reputation the officials framework (RMM) envisions to survey the irritated and furthermore sincere techniques for CPs and furthermore reflect this on their glory. on this paper, we recommend a energetic RMM within the united cloud with highlight on multi-residency. In a multi-inhabitant cloud, a patron depends upon the $\mathrm{CP}$ for reliable co-tenants. in this paper we recommend a one in every of a kind esteem the directors framework that encourages the CPs to choose extraordinary co-occupants to a higher than average consumer. Glory inside the Federated Cloud: A delivered together cloud is made by way of responsibilities from multiple cloud suppliers moreover, a digital framework request can be happy by means of more than one cloud service provider. In a consolidated cloud, a CP threats its protection assure recognition as it exhibits its blessings for diverse CPs (a computerized framework need may also degree over the advantages managed with the aid of multiple CPs). the problem in an online framework might also start from the physical assets controlled by means of various CPs. To have a look at the recognition of CPs, we will make use of the accompanying form of responses:1) remarks from CPs about numerous CPs: This form of protest is trying to realise as CPs needs to proportion facts with appreciate to their very own one in every of a type assets. 2) feedback from the customers regarding CPs: it is able to be even extra effectively procured. Be that as it is able to, such information might be malevolent and furthermore harmed. besides, as a web framework may additionally extend over the benefits had through multiple $\mathrm{CPs}$, it will baffle for a customer to exactly distinguish the $\mathrm{CP}$ that manages of a fault. three) remarks from the CPs regarding the customers: This country of evaluation is absolutely not testing to get. A CP can display the exercises of its clients and pick out whether a customer has horrendous goals. on this paper we utilize the 0.33 kind of contribution to assess the recognition of the CPs. it's far viable to mutilate such analysis. on this paper we endorse a framework that urges CPs to record ideal protest about the customers. CP's reputation and multi-residency: present RMMs for appropriated computer set up together contribution from customers and whole them to get lofty reputations for the CPs. also, 1) It dares to isolate between realistic protest from unwarranted criticism offered via the customers with respect to the execution of the CPs. 2) It moreover isolates between concerns in the physical frameworks and moreover the practical sporting activities of CPs that result in intrusion inside the physical framework. Thusly, deficiencies (which might be believed to be past the manage of the $\mathrm{CP}$ ) don't have an effect on reputations of CPs. In a stand proud of existing RMMs, on this paper we advocate a RMM with a consideration on multi-inhabitance. supplying computational property to others is the foremost strain of customers as 
different co-tenants is probably dangerous. be aware that,1) The co-tenants of a client are abnormal. on this manner, a patron can not pick with whom it will proportion

computational properties. 2) The patron confides within the $\mathrm{CP}$ to adjust super cotenants. via doing this, from a customer's point of view, with the emphasis on cotenancy,

it'll have a lot greater accept as true with in a $\mathrm{CP}$ at the off plausibility that it isolates amongst phenomenal and vindictive customers simply as on the off circumstance that it does not allow them to share homes. Thusly the restriction and practise of such partition among extraordinary and lethal clients is the critical parameter that picks the popularity of a $\mathrm{CP}$. on the occasion that a $\mathrm{CP}$ does not make such component up, it ought to get a reduced notoriety whilst differentiated just as another CP who makes such a component up. on this paper, we recommend a RMM that thinks approximately the CP's capability just as readiness to make such department among its clients. it's miles to the CP's little bit of leeway that it receives maximum distinguished utilization of its properties. on this manner, it lets in satisfactory co-residency self sufficient of the strategies for the clients. in this paper, we manipulate the delivered collectively cloud, where the bodily framework is contributed by diverse accomplices simply as it is a related diagram. inside the blended cloud, automated framework requests are mapped to the segments of the physical framework announced with the aid of diverse CPs. Thusly the CPs may match collectively to fulfill a automated framework want. observe that, 1) because the CPs group as much as acquire online framework wishes, it may take place that a CP, nation CP1, does no longer isolate among outstanding and detestable disapproved of customers regardless, a further $\mathrm{CP}$, nation $\mathrm{CP} 2$, does the backwards. at the off condition that $\mathrm{CP} 1$ what's extra, $\mathrm{CP} 2$ cooperate at that factor, in spite of the truth that $\mathrm{CP} 2$ does now not advocate, it would need to enable a first rate purchaser to turn into a co-inhabitant with a vile customer as a assets of this joint attempt with CP1. 2) therefore, the direct of a CP impacts its individuals. From this time ahead, we make the accompanying doubts: 1) CPs proportion the data regarding multi-inhabitance. furthermore this information can't be managed. 2) although, they'll deform the licensed lead of customers. rapidly, our RMM works as follows: 1) first of all, every CP distinguishes malevolent clients from notable clients and furthermore it ought to assign blessings for them with the final objective that the going with holds: an) It desires to no longer enable any sort of deadly consumer to wrap up a co-occupant of a decent client. b) it is able to allow unfavourable customers to percentage sources amongst themselves. 2) next, the CPs percentage records with appreciate to multitenancies. three) every $\mathrm{CP}$ reviews the lead of clients to the RMM.

\section{ASSOCIATED WORK}

The investigation in RMMs inside the IaaS angle of cloud enrolling is a mix of three research have a look at topics, (an) on-line esteem the officials, (b) recognition the board in scattered laptop just as (c) online framework introducing. in this discipline we hastily talk approximately the
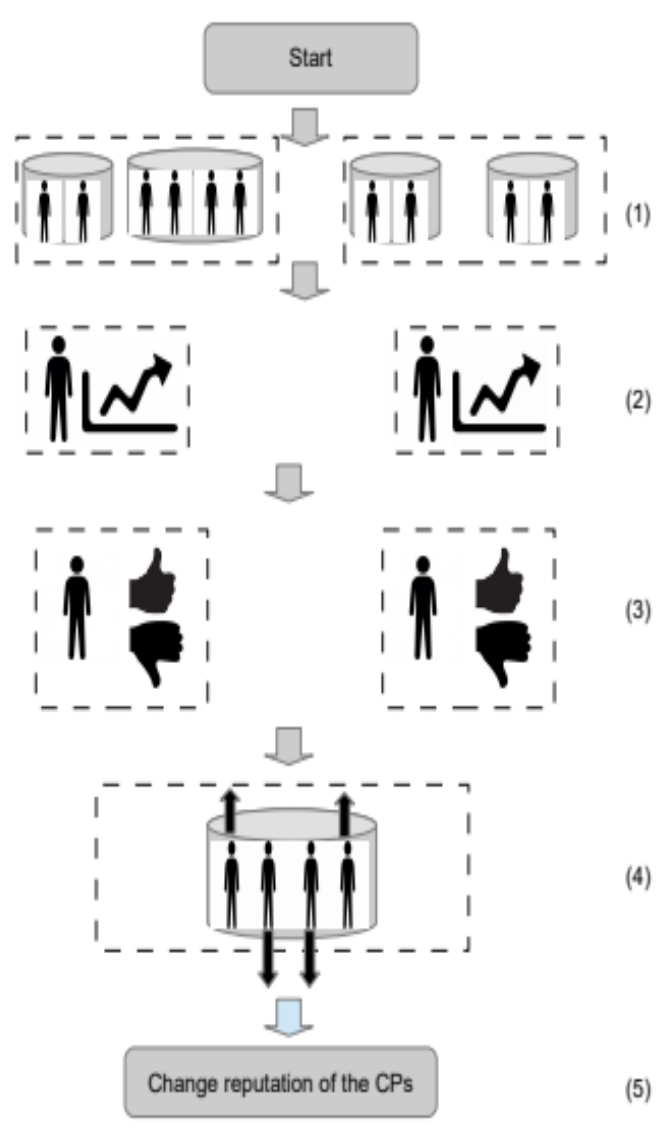

Fig. 1. The RMM is as follows:(1) each CP groups the users into sets of multi-tenants, (2) performance and activities of all users are monitored, (3) based on these observations the CPs vote (positive or negative) on each user and (4) finally, based on the change in the reputation of each user in a group the reputation of the CP's are changed.

exquisite in class in those take a look at out topics.As referenced in [1], there are 2 forms of gadgets to end up aware of out of line inputs. The endogenous systems [2] [3] in fact utilize the complaint to determine a wrong enter. the ones elements depend on legitimate residential houses of the inputs. On a normal basis these devices use the historic historical past of inputs and moreover anticipate that fundamental component of criticisms are low-priced. The exogenous elements join up with outer records to make your mind up if an input is cheap or unnecessary. events of such facts includes the believability of the customers. [4] makes use of customized comparability measure to rate the advice credibility. in this tool, the plausibility of an evaluator is managed with the aid of the usage of its pals whose have linked with it. Comparative technique to address determine the credibility is utilized in [5] [6] makes use of the administration count on as the parameter to make a decision the objection validity. however this device is powerless within the situations wherein the expert co-op faces competition and may ship unfounded objections regarding its competition. [7] recommends the heavy leading component calculation (WMA) that doles out masses within the form of manner that the family member weight allotted to the efficient publications is improved and also the member of the

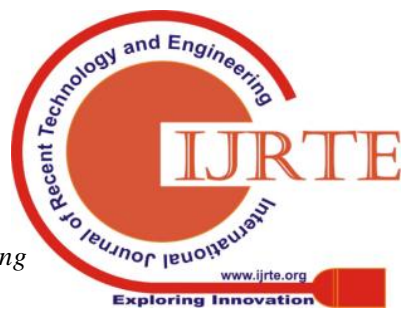


family weight administered to the unsuccessful overviews is decreased. [2] acknowledges the nearest next-door associates of a purchaser driving force primarily based mostly on their disposition similitude. Disposition distance is decided making use of the quantity of their comparative examinations for commonly assessed companies. within the wake of figuring out the closest buddies of the customer operator, group sifting is made use of to distinguish uncalled for ranking. [8] widens the notoriety the board frameworks superior in [9] to sift through uncalled for opinions the usage of the iterated dividing. [3] has used the customers notorieties in the remember of the venders reputation. [10] propose the TRAVOS display screen, that is a receive as true with fund and notoriety display for expert based totally online organizations. This trouble to start with determines the exactness of nowadays notoriety admonition based at the machine of specific and incorrect past guidelines which can be corresponding to the proper right here and now notoriety admonition. next, it modifications status exhortation based totally mostly on its accuracy. The thing of this responsibility is to limit the have an impact on of mistaken help. Be that as it can, this version count on that supplier drivers act reliably, which may not be legitimate plenty of the instant. There are more than one calculations for proceeding motivators for notoriety the board framework. [11] has honestly validated the foundation framework using an installment diversion to ensure that the specialists who offer honest inputs get more utility. [12] proposed an installment story for enter motels subsequent objection regarding a similar goal expert. The depends on detainees situation. in this model drivers with sincere objections beautify strength. [14] proposed a backing machine to achieve truthful objection. on this layout, in every alternate each activities offer a record about each different. on the off opportunity that the reviews in every change are not trusted then the 2 celebrations are rejected. [15] idea about the attainability of installation framework for inspiring honest criticisms for online closeout frameworks. [16] presents an iterative probabilistic approach for notoriety the board. In digital system dental implanting problem [17], a customer desires a gadget $\mathrm{GV}=(\mathrm{NV} \mathrm{EV}, \mathrm{WV})(\mathrm{NV}$ is the gathering of hubs, $\mathrm{EV}$ is the plan of edges, in addition to $\mathrm{WV}$ suggests the masses of the vertices and moreover the edges. The vertex weight shows the deal of CPU or computation utilization and heaps on the rims endorse the bandwidth for the communication networks) to be dental implanted proper into a physical gadget $\mathrm{G}=(\mathrm{N}, \mathrm{E}, \mathrm{W})(\mathrm{N}, \mathrm{E}, \mathrm{C}$ are hubs, elements and additionally thousands of the vertices and moreover the perimeters) with final touch aim that facts issues are happy (a) hundreds of the vertices and additionally the edges mapped into the bodily machine has to go to any charge the requested plenty of the vertices as well as the rims and additionally (b) for each and each requested vertex there should be one mapped vertex (or a hard and fast of vertices) and for every and each requested for facet there needs to be one mapped factor (or a mapped approach). take into account that a vertex (in addition to an part) can be shared through manner of one among a type VNEs. The VNE issue is understood to be NP-completed [18], [19], [20] and also that allows honest complaint. in this tool, a purpose pressure makes money if its criticism concerning an aim expert fits the motivating stress show display screen recommended in [13]

heristics are created in [21], [22], further to fee quote calculations are advanced in [23] VNE is a finances-friendly setup because it allows to lease a computational framework. notwithstanding the truth that, the inaccessibility of the physical tool due to troubles or resource distress the administrations depending on it. Survivable online system dental implanting (SVNE) [24] is a spread of the VNE problem wherein we similarly require to rent at the arrangement of enhancing properties inside the bodily device to the VNE plan. those superb assets comfy nonstop availability of the bodily belongings. The bodily device frustration may be single (or numerous) vertex as well as area frustration or both. [25] confirmed that $20 \%$ of all dissatisfaction is due to renovation, fifty $3 \%$ due to button related and $70 \%$ is due to unmarried connection disappointment. [26] confirmed that client interface sadness is multiple instances greater than hub dissatisfaction. There are techniques to address the SVNE issues [27] (a) tremendous techniques: in this technique we want to hold supports or non-obligatory belongings on ebook in case components of the physical system fizzles and additionally (b) receptive strategies: in this technique we want to discover the VNEs with the effects available possessions at the same time as elements of the physical device wreck down. at the same time as each actions do now not make certain information misfortune, the critical issue method is likewise confused over the innovation trouble of discovering the most charge effective reinforcementsts.

\section{EXPERIMENTAL ASSESSMENT \& RESULTS}

Casually the RMM is based totally on the following: 1) there can be a minimal type of CPs and also a restrained kind of customers. it is anticipated that each $\mathrm{CP}$ has digital arrange request from all customers. There are 3 sort of CPs, (a) levelheaded CP, (b) unreasonable CP and moreover (c) clever $\mathrm{CP}$. There are 2 types of clients, (a) excellent purchaser: one that does not set off any protection or on the other hand safety issues and additionally (b) pernicious client: one which creates safety and defense problems. A malicous cotenant would possibly adjust protection problems, for instance, side network attack (assault based on the physical implementation of the device), DOS attack [54], community examination attack [55] (assault to discover the geography of the gadget). We approve that if a CP has a purchaser then it may take a look at the client's bodily games likewise, view whether or not or now not it's far pernicious or not. 2) First, an) each $\mathrm{CP}$ names each patron as both an extraordinary customer or at the various different hand a malignant customer. b) It units aside virtual homes to the clients. c) The clients are parceled in gatherings with the cease goal that during every amassing all clients percentage assets with each specific, i.e., they are multi-occupant. d) every CP states sectors over the clients, i.e., they record the multi-occupancy statistics to the RMM. 3) next, CPs display exercises of the customers and document it to the RMM. A CP can either deliver a very good otherwise another time an negative vote in help of a customer. it is going to be ordinary that the combined cloud framework will 
supply the RMM with the methods for correspondence with the private CPs in addition to using such verbal exchange networks CPs frequently offer enter (i.e., favorable or bad ballot regarding the customers) to the RMM.A negative vote suggests that the patron is noxious as in line with the $\mathrm{CP}$ (that has given an terrible enact help of it) else it's miles a awesome customer. We make use of the adhering to communique show between the $\mathrm{CP}$ in addition to the clients: an) At each development, the customers create specific activities which is probably deciphered as symptoms of their splendid or evil-minded practices. b) At each progression, in the wake of gambling the celebrations created thru the clients, every $\mathrm{CP}$ evaluations the conduct of a consumer as pursues: - Favorable vote: It indicates that the CP sees the patron as a appropriate patron. - terrible ballot : It demonstrates that the $\mathrm{CP}$ sees the purchaser as a lethal consumer. c) After the RMM received the votes in preference of a purchaser, it ascertains the consumer's status for being goes after: I) If a patron obtains frequently favorable ballots than the terrible ballots after that its notoriety is increased. ii) If a customer gets more terrible ballots than the poor votes after that its status is decreased. iii) If a consumer obtains comparable kind of positive ballots in addition to the adverse ballots at that component its popularity keeps as inside the beyond. four) In every development, following freshening the repute of the clients, the RMM revitalizes the status of the CPs as seeks: a) for every birthday celebration of multi-inhabitant customers, if notoriety of all clients are broadened or fame of all customers are decreased at that issue, the CP's status is prolonged. b) For each and each accumulating of multi-occupant clients, if prestiges of a couple of customers are broadened (lessened) and additionally the same for some thing is still of the clients are decreased (broadened) at that element, the CP's notoriety is decreased. look at that, a CP's notoriety relies upon its right department of the clients, i.e., assigning the customers into activities (each celebration is a lot of multi-occupants, i.e., share belongings amongst themselves) wherein a suitable client need to stay in a party with without a doubt other exceptional clients and also a poisonous purchaser ought to live in a meeting with severa extraordinary terrible clients. The precision of a CP's branch of the clients is dictated through the modification of notoriety of the customers. In case it has virtually set as it were top notch clients in an occasion after that the notoriety of the customers in that birthday party will honestly increment (as numerous CPs enact desire of the dreadful and exquisite customers) and at the occasion that it has established surely horrible clients in a party then the notoriety of the clients in this event will virtually reduce. therefore a $\mathrm{CP}^{\prime}$ s reputation increments at the same time as the prestiges of all clients in an event either increment or decrease. yet, in case the $\mathrm{CP}$ has surely installation every the tremendous similarly to the awful customers in the equal lot after that the notoriety of more than one clients will increment in addition to the notoriety of diverse customers will clearly reduce. in the end, a CP's fame decreases when the prestiges of multiple customers in an occasion increment and prestiges of numerous clients within the equivalent collected lower. We represent numerous $n$ cloud vendors as $\mathrm{C}=\mathrm{c} 1, \ldots$, cn what is even extra, a whole lot of $\mathrm{m}$ customers as $\mathrm{U}=$. We approve the following: - Any kind of hassle constructing from a customer is diagnosed by way of manner of its host, i.e., the CP that has its on-line gadget.

- There may be an underlying notoriety of the customers, signified as $\mathrm{R}(\mathrm{ui}) \in[0,1]$. Notoriety zero demonstrates that the notoriety of the consumer is the base and notoriety 1 demonstrates that the notoriety of the purchaser is the best. All CPs recognise this underlying notoriety estimations of the clients.

- There is a uniform introductory notoriety for all CPs. Notoriety of the CPs is meant as $\mathrm{R}(\mathrm{ci}) \in \mathrm{R}>0$ (positive genuine number).

- If a client acts up at that point, its host should report such bad conduct to the RMM.

- Each CP produces a $\mathrm{k} \geq 1$ parcel over the clients, signified as $\pi=\{\pi 1, \ldots, \pi \mathrm{k}\}$. The arrangement of clients in each $\pi \mathrm{i}$ shares something like an edge or a vertex in the physical organize.

\section{Definition 1.}

(Virtual network embedding) Given virtual network request $G^{R}=\left(V^{r}, E^{R}\right)$, vertex weight function $W_{1}^{R}: V^{R} \rightarrow \mathrm{R}>0$ and edge Weight function $W_{2}^{R}: E^{R} \rightarrow \mathrm{R}>0$, a virtual network embedding f,maps $G^{R}$ to physical network $\mathrm{G}=(\mathrm{w}, \mathrm{E})$ with vertex weight function $\mathrm{W} 1: \mathrm{V} \rightarrow \mathrm{R}>0$ and edge weight function $\mathrm{W} 2: \mathrm{V} \rightarrow \mathrm{R}>\mathrm{O}$, such that the following holds:

1) For each vertex $v 1 \in V^{R}$ there Exits a subset $S \subset V$ such that $\mathrm{f}(\mathrm{V} 1)=\mathrm{S}$.

2) For each vertex v1 $\in V^{R}, W_{1}^{R}(\mathrm{v} 1) \leq$ $\sum V 2 \in \mathrm{f}(\mathrm{v} 1) \mathrm{W} 1(\mathrm{~V} 2)$.

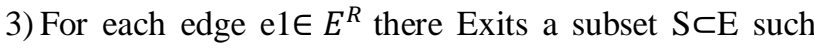
that $\mathrm{f}(\mathrm{v} 1)=\mathrm{S}$ and if $\mid \mathrm{S}>1$ then $\mathrm{S}$ is a converted path.

4) For each edge e1 $\in E^{R}, W_{2}^{R}(\mathrm{E} 1) \leq \sum e 2 \in \mathrm{f}(\mathrm{e} 1) \mathrm{W} 1(\mathrm{e} 1)$ Note that the virtual network embedding process does not offer computational resources exclusive to the users,rather they share computational resources.According to the patrician if two users share resources then they reside in the same group.

\section{Defination 2.}

(patrician over the user)the set of the user hosted by the $\mathrm{CP}$ ci Will be donated as $\mathrm{U}(\mathrm{ci}) \subset \mathrm{U}$ A patrician over $\mathrm{U}$ (ci) into $\mathrm{K} \geq 1$ sets will be donated as $\pi=\{\pi 1 \ldots . \pi \mathrm{k}\}$ such that the following holds:

- For each pair of users $\mathrm{U} 1$ and $\mathrm{U} 2$ in any set $\pi \mathrm{i}$ with virtual network requests $G^{1}=\left(V^{1}, E^{1}\right)$ and $G^{2}=\left(V^{2}, E^{2}\right)$ either $\mathrm{f}\left(V^{1}\right) \cap \mathrm{f}\left(V^{2}\right) \neq \varnothing$ or $\mathrm{f}\left(E^{1}\right) \cap \mathrm{f}\left(E^{2}\right) \neq \varnothing$ for any $\pi \mathrm{i} \cap \pi \mathrm{j} \neq \emptyset$ for $\mathrm{i} \neq \mathrm{j}$.

Now, we defind the user reputation as follows: 


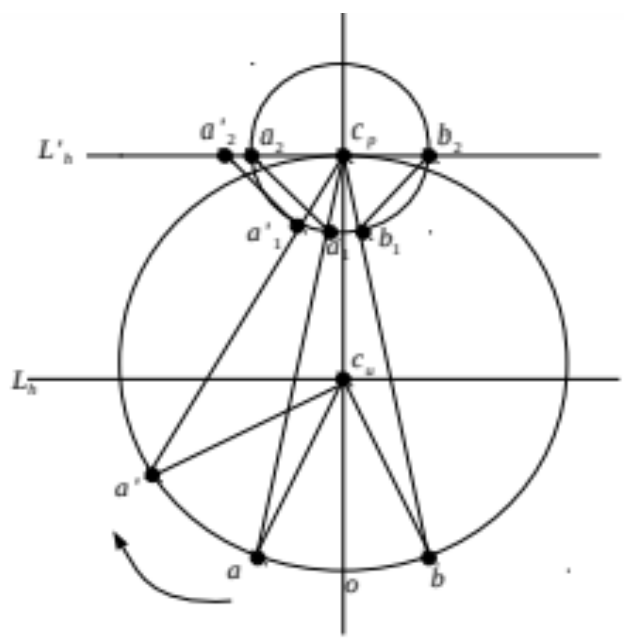

Fig (a)

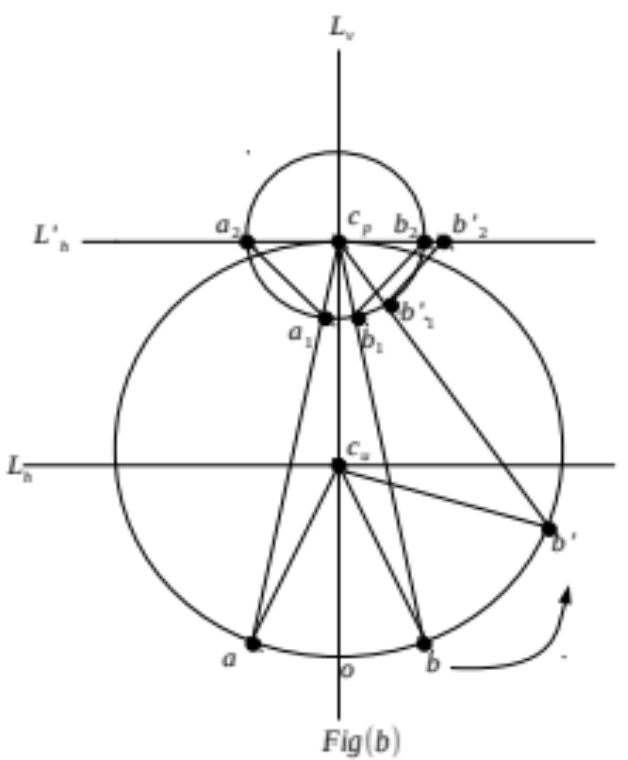

Fig. 4. Change in CP's reputation if it hosts one user.

Triangles $\Delta$ abcp and $\Delta \mathrm{acpz}$,

$\frac{a b}{a c p}=\sin \theta 1$

$\mathrm{xcp}=\mathrm{ab}=\mathrm{r} * \sin \theta 1$

$\mathrm{xz}=\mathrm{r}-\mathrm{r} * \sin \theta 1$

Also,

$\frac{c p b}{a c p}=\cos \theta 1$

In the triangle, $\Delta(\mathrm{xaz})$

$\tan \theta=\frac{x q}{x z}=\frac{r \cos \theta 1}{r-r * \sin \theta 1}=\frac{\cos \theta 1}{1-\sin \theta 1}$

In the triangle, $\Delta(\mathrm{a} 1, \mathrm{~b} 1, \mathrm{cp})$

$\cos \theta 2=\frac{y a 1}{r}, \mathrm{ya} 1=\mathrm{r} * \cos \theta 1$

$\tan \theta=\frac{r * \cos \theta 2 *(1-\sin \theta 1)}{y z 1}$

In the triangle, $\Delta(\mathrm{a} 1, \mathrm{~b} 1, \mathrm{cp})$

$\tan (90-\theta 2)=\frac{r * \cos \theta 2}{y c p}$

$\mathrm{Ycp}=\frac{r * \cos \theta 2}{\tan (90-\theta 2)}$

Hence

$$
\begin{aligned}
& \mathrm{Xy}=\mathrm{r}-\frac{r * \cos \theta 2}{\tan (90-\theta 2)} \\
& \mathrm{Zz} 1=\mathrm{yz} 1-\mathrm{yz} \\
& \mathrm{Zz} 1=\frac{r * \cos \theta 2 *(1-\sin \theta 1)}{\cos \theta 1}-\mathrm{r}+\frac{r * \cos \theta 2}{\tan (90-\theta 2)} \\
& \mathrm{Zz} 1=\frac{r * \cos \theta 2 *(1-\sin \theta 1)}{\cos \theta 1}-\mathrm{r}+\frac{r * \cos \theta 2 * \sin (\theta 2)}{\cos (\theta 2)} \\
& \mathrm{Zz} 1=\frac{r * \cos \theta 2 *(1-\sin \theta 1)}{\cos \theta 1}-\mathrm{r}+\mathrm{r} * \sin (\theta 2)
\end{aligned}
$$

Using lemma 1,in theorem 1 we prove that rational

CPs get better reputation than irrational and opportunistic CPs.

- There is a hidden notoriety of the clients, signified as $\mathrm{R}($ ui) $\in[0,1]$ Notoriety 0 demonstrates that the notoriety of the client is the base as well as prestige 1 demonstrates that the notoriety of the customer is the greatest. All CPs understand this underlying prestige evaluations of the customers.

- There is an uniform initial prestige for all CPs. Notoriety of the CPs is suggested as $\mathrm{R}$ ( ci) $\in \mathrm{R}>0$ (favorable real number).

- If a client breaks down at that point, its host needs to report such negative conduct to the RMM.

- Each CP generates a $k \geq 1$ parcel over the customers, signified as $\pi=\pi 1, \ldots, \pi \mathrm{k}$. The setup of clients in each $\pi \mathrm{i}$ shares something like an edge or a vertex in the physical organize.

\section{Meaning 1.}

( Digital network embedding) Given digital network request $\mathrm{G}^{\wedge} \mathrm{R}=\left(\mathrm{V}^{\wedge} \mathrm{r}, \mathrm{E}^{\wedge} \mathrm{R}\right)$, vertex weight feature $\mathrm{W}_{-} 1^{\wedge} \mathrm{R}$ : $\mathrm{V}^{\wedge} \mathrm{R} \rightarrow \mathrm{R}>0$ and also side Weight feature W_2 ${ }^{\wedge} \mathrm{R}: \mathrm{E}^{\wedge} \mathrm{R}$ $\rightarrow R>0$, a virtual network embedding f, maps $G^{\wedge} R$ to physical network $\mathrm{G}=(\mathrm{w}, \mathrm{E})$ with vertex weight feature $\mathrm{W} 1$ : $\mathrm{V} \rightarrow \mathrm{R}>0$ and edge weight function $\mathrm{W} 2: \mathrm{V} \rightarrow \mathrm{R}>\mathrm{o}$, such that the following holds:

For each vertex $\mathrm{v} 1 \in \mathrm{V} \wedge \mathrm{R}$ there Exits a part $\mathrm{S} \subset \mathrm{V}$ such that $\mathrm{f}(\mathrm{V} 1)=\mathrm{S}$.

For every vertex $\mathrm{v} 1 \in \mathrm{V}^{\wedge} \mathrm{R}, \mathrm{W}_{-} 1^{\wedge} \mathrm{R}(\mathrm{v} 1) \leq \sum$ 亚 $\mathrm{V} 2 \in \mathrm{f}($ v1) W1( V2).

For each and every edge $\mathrm{e} 1 \in \mathrm{E}^{\wedge} \mathrm{R}$ there Leaves a part $\mathrm{S} \subset$ E such that $f(v 1)=S$ and if $\mid S>1$ then $S$ is a modified course.

For each and every edge e $1 \in \mathrm{E}^{\wedge} \mathrm{R}, \mathrm{W} 2^{\wedge} \mathrm{R}(\mathrm{E} 1) \leq \sum$ 器: $\mathrm{e} 2 \in \mathrm{f}(\mathrm{e} 1) \mathrm{W} 1$ ( e1) Keep in mind that the digital network embedding procedure does not use computational sources unique to the customers, instead they share computational resources.According to the patrician if 2 individuals share sources after that they reside in the very same group.

\section{Defination 2.}

(patrician over the customer) the collection of the individual organized by the $\mathrm{CP}$ ci Will certainly be donated as $\mathrm{U}(\mathrm{ci}) \subset \mathrm{U}$ A patrician over $\mathrm{U}$ ( ci) right into $\mathrm{K} \geq 1$ sets will certainly be contributed as $\pi=\pi 1 \ldots \pi \mathrm{k}$ such that the adhering to holds:

For every set of customers U1 and also U2 in any kind of established $\pi \mathrm{i}$ with online network requests $\mathrm{G}^{\wedge} 1=\left(\mathrm{V}^{\wedge} 1, \mathrm{E}^{\wedge}\right.$ 1) and also $\mathrm{G}^{\wedge} 2=\left(\mathrm{V}^{\wedge} 2, \mathrm{E}^{\wedge} 2\right)$ either $\mathrm{f}\left(\mathrm{V}^{\wedge} 1\right) \cap \mathrm{f}\left(\mathrm{V}^{\wedge} 2\right) \neq$ $\emptyset$ or $\mathrm{f}\left(\mathrm{E}^{\wedge} 1\right) \cap \mathrm{f}\left(\mathrm{E}^{\wedge} 2\right) \neq \emptyset$ for any type of $\pi \mathrm{i} \cap \pi \mathrm{j} \neq \varnothing$ for $\mathrm{i} \neq$ j.

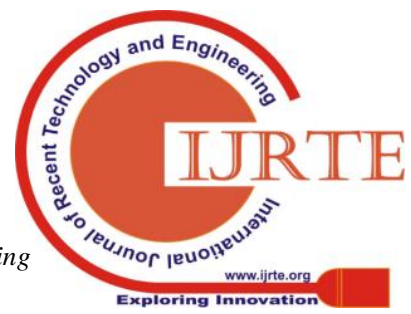


Currently, we defind the customer reputation as complies with:

Triangulars $\Delta$ abcp and also $\Delta$ acpz,

$\mathrm{ab} / \mathrm{acp}=$ wrong $\theta 1$.

$\mathrm{xcp}=$ abdominal $=\mathrm{r} * \sin \theta 1$.

$\mathrm{xz}=\mathrm{r}-\mathrm{r} *$ wrong $\theta 1$.

Likewise,

$\mathrm{cpb} / \mathrm{acp}=\cos \theta 1$.

$\tan \theta=\mathrm{xq} / \mathrm{xz}=(\mathrm{r} \cos \theta 1) /(\mathrm{r}-\mathrm{r} *$ transgression $\theta 1)=\cos \theta 1 /($

$1-\sin \theta 1)$.

In the triangle, $\Delta(\mathrm{a} 1, \mathrm{~b} 1, \mathrm{cp})$.

$\cos \theta 2=$ ya $1 / \mathrm{r}, \mathrm{ya} 1=\mathrm{r} * \cos \theta 1$.

$\tan \theta=(r * \cos \theta 2 *(1-\sin \theta 1)) / y z 1$.

$\tan (90-\theta 2)=(r * \cos \theta 2) / \mathrm{ycp}$.

$\mathrm{Ycp}=(\mathrm{r} * \cos \theta 2) / \tan (90-\theta 2)$.

Thus.

$X y=r-(r * \cos \theta 2) / \tan (90-\theta 2)$.

$\mathrm{Zz} 1=\mathrm{yz} 1-\mathrm{yz}$.

$\mathrm{Zz} 1=(\mathrm{r} * \cos \theta 2 *(1-\sin \theta 1)) / \cos \theta 1-\mathrm{r}+(\mathrm{r} * \cos \theta 2) / \tan ($ 90- $\theta 2)$.

$\mathrm{Zz} 1=(\mathrm{r} * \cos \theta 2 *(1-\sin \theta 1)) / \cos \theta 1-\mathrm{r}+(\mathrm{r} * \cos \theta 2 *$ transgression $(\theta 2)) / \cos (\theta 2)$.

$\mathrm{Zz} 1=(\mathrm{r} * \cos \theta 2 *(1-\sin \theta 1)) / \cos \theta 1-\mathrm{r}+\mathrm{r} * \operatorname{transgression}($ $\theta 2)$.

Making use of lemma 1 , in theory 1 we verify that logical.

CPs improve credibility than irrational as well as opportunistic CPs.

\section{CONCLUSION}

Co-occupancy makes cloud pc price amplify charming anyway it furthermore offers new hazard from noxious co-population. A benefactor depends at the $\mathrm{CP}$ for designation of peril unfastened co-occupants. Our goal in this paper is to make a RMM that energizes CPsto make proper office amongst legitimate and pernicious clients, i.e., an outstanding consumer receives exceptional particular incredible individuals as cotenants. the present RMMs for cloud laptop do never once more remember this stipulations to evaluate believability of the CPs. the prevailing RMMs for cloud laptop make use of customary social occasion of feedback from customers to expense the CPs. on this paper we've got really set up an wonderful RMM that urges CPs to isolate in among exquisite and ruinous customers and assign resources in the form of way that they don't price property.using coherent simply as theoretical exams we show the rightness of the energized RMM.

\section{REFERENCES}

1. A. Whitby, A. Jsang, and additionally J. Indulska, "Sifting through out of line scores in bayesian melody file systems," in AAMAS04, 2004.

2. C. Dellarocas, "Immunizing at the internet notoriety pronouncing structures towards shameful scores and furthermore unjust conduct," in grievances of the second one ACM meeting on computerized enterprise task, ser. EC '00. big apple, massive apple, u.s.: ACM, 2000, pp. 100 and fifty- -157.

3. M. Chen and J. P. Singh, "computer just as the use of notorieties for net value determinations," in techniques of the $0.33 \mathrm{ACM}$ get collectively on electronic commercial
In the triangular, $\Delta(\mathrm{xaz})$.

In the triangular, $\Delta(\mathrm{a} 1, \mathrm{~b} 1, \mathrm{cp})$.

enterprise, ser. EC '01. the big apple city, new york, US of the usa: ACM, 2001, pp. 154- - 162.

4. A. Das and furthermore M. Islam, "Securedtrust: A dynamic anticipate calculation rendition for shielded association in multiagent frameworks," clean notwithstanding loosened up Computing, IEEE Purchases on, vol. nine, no. 2, pp. 261- - 274, March 2012.

5. A. o.k.. Despotovic Z, "high-quality excellent stage likelihood gauge of companions? exhibitions in $\mathrm{p} 2 \mathrm{p}$ systems," in techniques of the second workshop on the monetary components of allotted frameworks, 2004.

6. S. D. Kamvar, M. T. Schlosser, and H. Garcia-Molina, "The eigentrust calculation for on-line acknowledgment manage in $p 2 p$ structures," in methods of the twelfth ordinary Seminar on net, ser. WWW '03. big apple, ny, americaa.: ACM, 2003, pp. 640- - 651.

7. B. Yu, M. Singh, and moreover very well. Sycara, "developing rely on massive scale distributed structures," in Multi-Agent assurance notwithstanding Survivability, 2004 IEEE First Symposium on, Aug 2004, pp. 1- - 10.

8. A. Whitby, A. Jsang, and moreover J. Indulska, "Stressing unjustifiable ratings in bayesian prevalence structures," 2004.

9. B. E. endeavor, A. Jsang, and R. Ismail, "The beta validity machine," In structures of the fifteenth Hemorrhaged computerized task meeting, 2002.

10. W. T. L. Teacy, J. Patel, N. R. Jennings, and also M. success, "looking after misinformed tune record assets: Experimental assessment of a probabilistic rely on version," in machine of the Fourth global Joint Seminar on self-sufficient operators and Multiagent structures, ser. AAMAS '05. the big apple, big apple, joined statesA.: ACM, 2005, pp. 997- - 1004.

11. H. Zhao, X. Yang, and furthermore X. Li, "A praise framework to aid actual reports in online acknowledgment frameworks," J. Netw. Comput. Appl., vol. 35, no. 3, pp. 951- - 961, might also 2012.

12. R. Jurca notwithstanding B. Faltings, "An concept reasonable on-line notoriety instrument," in methodologies of the Secondly regular Joint accumulating on self-governing experts and also Multiagent arrangements, ser. AAMAS '03. large apple city, ny, u.s.: ACM, 2003, pp. 1026- - 1027.

13. M. Feldman, ok. Lai, I. Stoica, and J. Chuang, "strong inspiration methodologies for distributed systems," in strategy of the 5th ACM Seminar on advanced undertaking, ser. EC '04. big apple city, big apple, US of americaA.: ACM, 2004, pp. 102- - 111.

14. T. G. Papaioannou notwithstanding G. D. Stamoulis, "A motivating forces' apparatus advancing true remarks in shared systems," in method of the 5th IEEE overall Seminar on Cluster laptop and the Grid - quantity 01, ser. CCGRID '05. Washington, DC, US of americaA.: IEEE pc framework lifestyle, 2005, pp. 275- - 283.

15. J. Witkowski, "valid input for favoring believability structures," CoRR, vol. abs/1203.3527, 2012.

16. E. Ayday just as F. Fekri, "strong popularity manage the usage of probabilistic message going," in claims of the general Communications collecting, GLOBECOM 2011, five-9 December 2011, Houston, Texas, US, 2011, pp. 1- 5.

17. Y. Xin, I. Baldine, A. Mandal, C. Heermann, J. Pursue, however A. Yumerefendi, "introducing digital topologies in organized mists," in tactics of the 6th international Seminar on future internet advancements, ser. CFI 'eleven. big apple city, big apple, US: ACM, 2011, pp. 26- - 29.

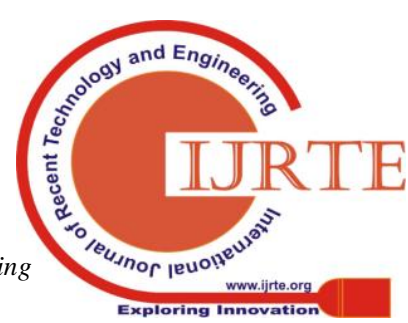


18. Y. Zhu and moreover M. Ammar, "Calculations for assigning substrate network assets to digital gadget components," in INFOCOM 2006. 25th IEEE worldwide accumulating on laptop Communications. technique, April 2006, pp. 1- - 12

19. [19] D. G. Andersen, "Hypothetical techniques to hub venture," 2002.

20. J. Lischka simply as H. Karl, "A virtual network mapping recipe essentially depending on subgraph isomorphism disclosure," in method of the vital ACM Workshop on Virtualized Framework solutions and moreover Architectures, ser. VISA '09. new york town, new york, united states of americaa.: ACM, 2009, pp. eighty one- 88.

21. M. Yu, Y. Yi, J. Rexford, and M. Chiang, "Reevaluating digital network inserting: Substratum help for way part and moreover motion," SIGCOMM Comput. Commun. Rev., vol. 38, no. 2, pp. 17- - 29, Mar. 2008.

22. [22] R. Ricci, C. Alfeld, notwithstanding J. Lepreau, "A solver for the system testbed mapping difficulty," SIGCOMM Comput. Commun. Rev., vol. 33, no. 2, pp. sixty five- - eighty one, Apr. 2003.

23. N. Chowdhury, M. Rahman, and furthermore R. Boutaba, "automated network putting in with composed hub just as net link mapping," in INFOCOM 2009, IEEE, April 2009, pp. 783- - 791.

24. X. A. Sandra Herker, Ashiq Khan, "observe on survivable advanced gadget putting in inconvenience and moreover contributions," ICNS 2013: The 9th accepted Seminar on Networking and administrations, 2013.

25. A. Markopoulou, G. Iannaccone, S. Bhattacharyya, C.- N Chuah, notwithstanding C. Diot, "Portrayal of failings in an ip backbone," in INFOCOM 2004. Twenty-0.33 AnnualJoint display of the IEEE computer device and furthermore Communications Societies, vol. four, March 2004, pp. 2307- - 2317 vol.four.

26. P. Gill, N. Jain, and also N. Nagappan, "perceiving system failings in realities focuses: length, evaluation, and influences," SIGCOMM Comput. Commun. Rev., vol. 41, no. four, pp. 350- - 361, Aug. 2011.

27. S. Ramamurthy, L. Sahasrabuddhe, and B. Mukherjee, "Survivable wdm work structures," Lightwave Innovation, mag of, vol. 21, no. four, pp. 870- - 883, April 2003. 